\title{
Rupture of tibialis anterior associated with a closed midshaft tibial fracture
}

\author{
Robert Din, L Therkilsden
}

A 41 year old man presented after a road traffic accident. He was a motorcyclist travelling at $30 \mathrm{mph}$ and was thrown from his motorcycle. Physical examination was unremarkable apart from a painful deformed right lower limb, with neurovascular structures intact.

Radiography revealed a midshaft oblique fracture of the right tibia and fibula, with normal knee and ankle joints, as shown in fig 1.

The midshaft tibia fracture was subsequently treated with a Russel Taylor interlock-

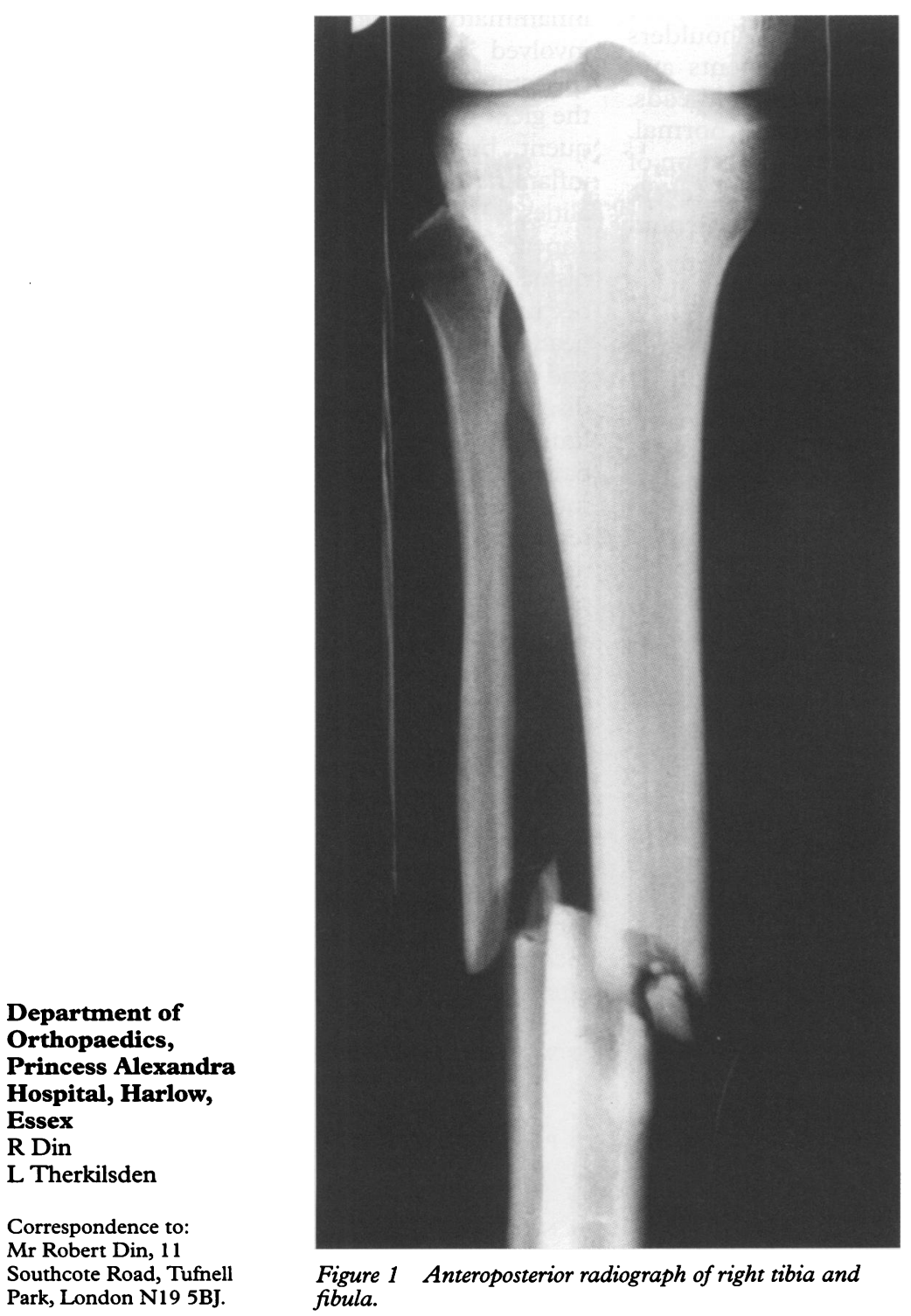

ing tibial nail, a closed operative procedure. The patient made an unremarkable recovery and was discharged home five days postoperatively.

The patient attended the outpatient clinic two weeks after hospital discharge with a complaint of inability to actively dorsiflex his right ankle. Physical examination revealed a palpable gap in the soft tissues over the anterior aspect of the right tibia midshaft, reduced power of dorsiflexion of the right foot, and a neurovascular intact lower limb.

The defect in the soft tissues over the right tibia was surgically explored and at operation the tibialis anterior was found ruptured.

Acute rupture of the tibialis anterior is an uncommon injury and has been rarely reported in the literature; it was first described by Burman. ${ }^{1}$ If this injury is missed it has the potential to cause weakness of the ankle and pain in the lower limb. ${ }^{2}$ It usually occurs in the elderly population, where disruptions occur through abnormal tendons. Lapidius ${ }^{3}$ described aseptic necrosis and Moberg described mucoid degeneration in tendons of the elderly, resulting in rupture. ${ }^{4}$ Tibialis anterior rupture has also been associated with diabetes mellitus, hypothyroidism, gout, and local steroid injection.

This case is unique in that the tibialis anterior disruption had been caused by a closed tibial shaft fracture. It is a difficult diagnosis to make, unless dorsiflexion of the ankle is tested for against resistance and compared with the uninvolved side, in the acutely injured patient, preferably in the accident and emergency department at initial presentation The action of extensor digitorum longus and extensor hallucis longus, which produce concomitant ankle dorsiflexion, may hamper the diagnosis of tibialis anterior rupture.

This case report illustrates an unusual complication of a tibial shaft fracture, and the need for a full physical examination at the time of presentation, if this injury is not to be missed.

1 Burman MS. Subcutaneous rupture of the tendon of the tibialis anticus. Ann Surg 1934;100:368-72.

2 Dooley BJ, Kudelka P, Menelaus MB, et al. Subcutaneous rupture of the tibialis anterior. Fournal of foint Surgery 1980;62B:471-2.

3 Lapidius PW. Indirect subcutaneous rupture of the anterior tibial tendon. Bulletin of Hospital for foint Disease and Orthopaedic Institute 1941;2:119-27.

4 Moberg E. Subcutaneous rupture of the tendon of tibialis anterior or muscle. Acta Chirurgica Scandinavica 1947;95: 455-60. 